(2.16) $\mathrm{mm})$. The reduction was statistically different versus placebo for both doses (400 mg p < $0.0001 ; 95 \% \mathrm{Cl},(-19.95$ to -6.04$)$ and $200 \mathrm{mg} \mathrm{p}=0.0008 ; 95 \% \mathrm{Cl},(-17.64$ to -4.12$)$. There was no effect on the WOMAC subscale of pain.

Conclusion: Rubus idaeus leaf extract, even at the low dose of $200 \mathrm{mg}$, was effective to relieve pain at short-term in-patient with knee OA. As Rubus idaeus leaf extract is well tolerated, it could be an alternative to nonsteroidal anti-inflammatory drugs and paracetamol to relieve knee joint pain in OA patients. REFERENCES:

[1]Bannuru RR, Osani MC, Vaysbrot EE, Arden NK, Bennell K, Bierma-Zeinstra SMA, Kraus VB, Lohmander LS, Abbott JH, Bhandari M, Blanco FJ, Espinosa R, Haugen IK, Lin J, Mandl LA, Moilanen E, Nakamura N, Snyder-Mackler L, Trojian T, Underwood $\mathrm{M}$, McAlindon TE. OARSI guidelines for the non-surgical management of knee, hip, and polyarticular osteoarthritis. Osteoarthritis Cartilage. 2019 Nov;27(11):1578-1589. Disclosure of Interests: Yves Henrotin Speakers bureau: Expanscience, Nestlé, Tilman, Consultant of: Naturex SA, Artialis SA, Tilman SA, Seigakaku, Stemmatters, Expanscience, Romain Le Cozannet Employee of: Naturex SA, Pascale Franca-Berthon Employee of: Naturex SA, Romain Truillet Employee of: Atlanstat, Martine Cohen Solal: None declared

DOI: 10.1136/annrheumdis-2021-eular.1493

\section{POS0282 1 EVALUATION OF A NEW HYALURONATE FORMULATION ONE YEAR AFTER SINGLE INJECTION TO PATIENTS WITH SYMPTOMATIC KNEE OSTEOARTHRITIS (SOYA STUDY)}

C. Gavín ${ }^{1}$, F. Moreno ${ }^{1}$, F. J. Blanco ${ }^{2}$, J. L. Pablos ${ }^{3}$, M. A. Caracuel-Ruiz ${ }^{4}$, J. Rosas ${ }^{5}$, P. Hernández-Esteban ${ }^{6}$, F. Navarro ${ }^{7}$, P. Coronel ${ }^{8}$, M. Gimeno ${ }^{9}$ on behalf of SOYA study group. ${ }^{1}$ H.U. Fundación Alcorcón, Traumatology, Alcorcón (Madrid), Spain; ${ }^{2}$ C.H.U. a Coruña, Rheumatology, A Coruña, Spain; ${ }^{3}$ H.U. 12 de Octubre, Rheumatology, Madrid, Spain; ${ }^{4}$ H.U. Reina Sofía, Rheumatology, Córdoba, Spain ${ }^{5}$ H. Marina Baixa, Rheumatology, Villajoyosa (Alicante), Spain; ${ }^{6}$ H.U. La Paz, Traumatology, Madrid, Spain; ${ }^{7}$ H.G.U. de Elche, Rheumatology, Elche (Alicante), Spain; ${ }^{8}$ Meiji Pharma Spain, S.A., D. International, Alcalá de Henares (Madrid), Spain; ${ }^{9}$ Meiji Pharma Spain, Clinical Development, Alcala de Henares, Spain

Background: Osteoarthritis $(O A)$ is a leading cause of chronic pain and disability, and its prevalence is expected to increase worldwide (1) being the knee the most affected joint, especially in older adults. Intra-articular hyaluronic acid (HA) could be of particular benefit in OA patients with co-morbidities, and in case of inadequate response to other pharmacological treatments (2). HA has been generally administered in cycles of 3-5 injections, however, due to the pressure on public health systems, the trend is to reduce the number of injections maintaining the duration of effects as longer as possible.

Objectives: To assess the effectiveness and safety of a new formulation of HA, up to one year after one single injection to patients in the SOYA (Symptomatic Osteoarthritis one Year Assessment) study.

Methods: Patients with Kellgren-Lawrence $(\mathrm{KL})$ grade 2-3 and Visual Analogue Scale (VAS) pain $>=40-<80 \mathrm{~mm}$ were prospectively included to receive a single injection of MPS-HA2\%. At $6 \mathrm{~m}$ a second injection could be offered to selected patients. Primary outcome was reduction of VAS pain in the target knee. VAS for joint pain and WOMAC were recorded at 6 and 12m; Minimally Clinical Important Improvement MCII ( $>=20 \%$ relative change for VAS pain) and patient and investigator assessments (PGA, IGA) (Likert scale 0-4 points) were also estimated. Adverse events were recorded for safety assessment.

Results: One hundred and one patients (mean age 68 years, $74 \%$ female and $78 \%$ with overweight) were included. Mean pain at baseline in the target knee was $63.57 \mathrm{~mm}$ and $57 \%$ were grade $3 \mathrm{KL}$ with a mean evolution of 7.5 years. Table 1 shows the improvement in VAS and WOMAC scores at 6 and 12 months in the mITT population. Similar results were obtained in PP population.

Table 1. Changes in VAS and WOMAC scores, expressed as mean values

\begin{tabular}{lccccc}
\hline SCORE & Baseline & \multicolumn{3}{c}{6 months } & \multicolumn{1}{c}{12 months } \\
\cline { 2 - 6 } & value & value & $\begin{array}{c}\% \text { variation } \\
(95 \% \mathrm{Cl})\end{array}$ & value & $\begin{array}{c}\% \text { variation } \\
(95 \% \mathrm{Cl})\end{array}$ \\
\hline $\begin{array}{l}\text { VAS pain } \\
\text { WOMAC }\end{array}$ & 63.57 & 37.59 & $-39.74^{\star}(-49.23 ;-30.25)$ & 38.37 & $-37.67^{\star}(-47.82 ;-27.52)$ \\
total & 50.19 & 31.88 & $-37.96^{\star}(-46.83 ;-29.09)$ & 31.65 & $-36.47^{\star}(-46.20 ;-26.73)$ \\
pain & 49.37 & 32.01 & $-32.92^{*}(-43.37 ;-22.46)$ & 31.08 & $-32.07^{\star}(-43.19 ;-20.95)$ \\
stiffness & 49.12 & 28.35 & $-35.24^{\star \star}(-53.22 ;-17.23)$ & 28.71 & $-34.08^{\star}(-49.30 ; 18.86)$ \\
function & 52.07 & 35.30 & $-33.95^{\star}(-43.49 ;-24.40)$ & 35.15 & $-32.71^{\star}(-42.80 ;-22.62)$
\end{tabular}

${ }^{*}$ p-value: $0.0001 ;{ }^{* *}$-value: 0.0002 . Student test

The MCll was achieved by $66.3 \%$ of patients at $6 \mathrm{~m}$ and $62.2 \%$ at $12 \mathrm{~m}$. Regarding PGA mean score was 2.44 at baseline, 1.35 at $6 \mathrm{~m}$ and 1.46 at $12 \mathrm{~m}$ (Wilcoxon, $\mathrm{p}$-value $<0.05)$. As for the IGA mean score was 2.29 at baseline, 1.06 at $6 \mathrm{~m}$ and 1.48 at $12 \mathrm{~m}$ (Wilcoxon, p-value $<0.05$ ).
Fourteen patients received a second injection at $6 \mathrm{~m}$ and $50 \%$ of them achieved at $12 \mathrm{~m}$ a significant and clinically relevant improvement compared to baseline, above the $20 \%$ established for the MCII.

In total, 12 adverse events (8 patients) were reported, all of them local, non-serious, and of mild-moderate intensity.

Conclusion: Viscosupplementation with a single intra-articular injection of MPS-HA2\% has proven to be effective and well tolerated up to 12 months after treatment. The re-infiltration of the joint in appropriate cases has proven to be effective in a significant number of patients. The acceptability of the treatment by the patient was optimal.

REFERENCES:

[1] Sebbag E, Felten R, Sagez F, et al. The world-wide burden of musculoskeletal diseases: a systematic analysis of the World Health Organization Burden of Diseases Database. Ann Rheum Dis. 2019 Jun;78(6) 844-848

[2] Bruyère $\mathrm{O}$, Cooper $\mathrm{C}$, Pelletier JP, et al. An algorithm recommendation for the management of knee osteoarthritis in Europe and internationally: a report from a task force of the European Society for Clinical and Economic Aspects of Osteoporosis and Osteoarthritis (ESCEO). Semin Arthritis Rheum. 2014 Dec;44(3):253-63.

Disclosure of Interests: Carlos Gavín: None declared, Francisco Moreno: None declared, Francisco J. Blanco: None declared, José Luis Pablos: None declared, Miguel A. Caracuel-Ruiz: None declared, Jose Rosas: None declared, Pablo Hernández-Esteban: None declared, Francisco Navarro: None declared, PILAR CORONEL Employee of: Meiji Pharma Spain, S.A., Mercedes Gimeno Employee of: Employee of Meiji Pharma Spain, S.A.

DOI: 10.1136/annrheumdis-2021-eular.1037

\section{\begin{tabular}{|l|l|l|}
\hline POS0283 TREATMENT PATTERNS AND CLINICAL \\
\hline
\end{tabular} CHARACTERISTICS OF PATIENTS WITH OSTEOARTHRITIS OF THE HIP AND/OR KNEE TREATED WITH TRADITIONAL NSAIDS VS COX-2S: A REAL-WORLD STUDY OF COMMERCIALLY-INSURED PATIENTS}

S. Silverman ${ }^{1,2}$, P. Schepman ${ }^{3}$, J. B. Rice ${ }^{4}$, C. Beck ${ }^{3}$, A. White ${ }^{4}$, S. Thakkar $^{3}$ M. Johnson ${ }^{4}$, R. Robinson ${ }^{5}$, B. Emir ${ }^{3} .{ }^{1}$ Cedars Sinai Medical Center, Los Angeles, United States of America; ${ }^{2}$ David Geffen School of Medicine at UCLA, Los Angeles, United States of America; ${ }^{3}$ Pfizer, New York, United States of America; ${ }^{4}$ Analysis Group, HEOR, Boston, United States of America; ${ }^{5}$ Eli Lilly and Company, Indianapolis, United States of America

Background: The 2019 American College of Rheumatology (ACR) guidelines strongly recommend oral nonsteroidal anti-inflammatory drugs (NSAIDs) for management of hip and knee osteoarthritis $(\mathrm{OA})$ and strongly recommend topical NSAIDs for knee OA. There are, however, important safety considerations with NSAIDs in terms of increased rates of gastrointestinal, cardiovascular, and renal events. Given these risks, it is important to understand the characteristics and drug utilization of the patients who start treatment on these different treatments (i.e., traditional NSAIDs [tNSAIDs] and cyclooxygenase-2 inhibitors [COX-2s]).

Objectives: The goal of this research was to describe and compare baseline characteristics of commercially-insured patients diagnosed with $O A$ of the hip and/or knee who started treatment on different types of NSAIDs (i.e., ora tNSAIDs, topical tNSAIDs, and COX-2s).

Methods: The Optum Healthcare Solutions, Inc. claims database (1/2012$3 / 2017$ ) was used to identify patients $\geq 18$ years old, with $\geq 2$ diagnoses of hip and/or knee OA, and $\geq 90$ days supply of oral tNSAIDs, topical tNSAIDs, or COX-2s during the one-year follow up period. The index date was defined as the first prescription after the first OA diagnosis. Patients were assigned to cohorts based on the type of NSAID prescribed on index date. Patients were required to be continuously-enrolled six months before (baseline period) and 36 months after (follow-up period) the index date. Demographic and clinical characteristics including age, sex, comorbidities, and healthcare resource use (HRU) were summarized during baseline. Drug utilization characteristics including days supply and number of prescriptions for the different NSAIDs types were summarized during follow-up period.

Results: Data for 23,796 patients were analyzed: 18,100 patients received oral tNSAIDs, 4,825 received COX-2s, and 871 topical tNSAIDs. Patients who initiated treatment on oral tNSAIDs were the youngest (mean age of 60.6 vs. 64.6 for COX-2s and 65.0 for topical tNSAIDs) and topical tNSAIDs had the highest proportion of female patients ( $71 \%$ vs. $62 \%$ for oral tNSAIDs and $63 \%$ for COX-2s). The topical tNSAIDs cohort had the highest presence of chronic kidney disease $(2.6 \%$ vs. $1.0 \%$ and $1.5 \%$ for oral tNSAIDs and COX-2s, respectively) and congestive heart failure $(2.5 \%$ vs. $0.8 \%$ and $1.7 \%$ for oral tNSAIDs and COX-2s, respectively) at baseline. In terms of HRU during baseline, topical 
tNSAIDs had the most patients with emergency department visits $(20.8 \%$ vs. $16.7 \%$ in both COX-2s and oral tNSAIDs), and COX-2 had the most patients with inpatient visits (18.1\% vs. $15.4 \%$ for topical tNSAIDs and $11.8 \%$ for oral tNSAIDs). Oral tNSAIDs had the lowest total all-cause cost $(\$ 6,504)$, and the topical tNSAIDs cohort had the highest costs $(\$ 8,455)$, but fairly comparable with COX-2s $(\$ 8,289)$. During follow-up, oral tNSAIDs patients stayed mostly on oral tNSAIDs as less than $15 \%$ of oral tNSAIDs patients later had a prescription for COX-2s or topical tNSAIDs. $37 \%$ of COX-2 patients and $56 \%$ of topical tNSAIDs patients later took oral tNSAIDs. Topical tNSAIDs patients had an average of 184.4 days of supply for topical tNSAIDs yet also extensively used oral NSAIDs during follow-up (average days of supply for oral tNSAIDs was 315.5 days and for COX-2s was 383.5 days)

Conclusion: This study suggests that patients with more complex comorbidity profiles, including higher rates of adverse effects, often start pharmacological treatment with topical tNSAIDs. However, patients who start treatment with topical tNSAIDs switch to other types of NSAIDs; oral tNSAIDs were the most frequently prescribed treatment across the cohorts. Thus, despite the safety concerns with oral tNSAIDs and COX-2s, patients are still placed on these treatments to manage their OA pain. There is a need for new innovative treatments as there is currently a lack of other options.

Disclosure of Interests: Stuart Silverman Consultant of: Stuart Silverman is a paid consultant to Pfizer and Eli Lilly and Company in connection with this study, Patricia Schepman Shareholder of: Patricia Schepman is an employee of Pfizer with stock and/or stock options, Employee of: Pfizer, James B Rice Consultant of: Brad Rice is an employee of Analysis Group, who were paid consultants to Pfizer and Eli Lilly and Company for this study, Craig Beck Shareholder of: Craig Beck is an employee of Pfizer with stock and/or stock options, Employee of: Pfizer, Alan White Consultant of: Alan White is an employee of Analysis Group, who were paid consultants to Pfizer and Eli Lilly and Company for this study, Sheena Thakkar Shareholder of: Sheena Thakkar is an employee of Pfizer with stock and/or stock options, Employee of: Pfizer, Michaela Johnson Consultant of: Michaela Johnson is an employee of Analysis Group, who were paid consultants to Pfizer and Eli Lilly and Company for this study, Rebecca Robinson Shareholder of: Rebecca Robinson is an employee and minor stockholder of Eli Lilly and Company, Employee of: Eli Lilly and Company, Birol Emir Shareholder of: Birol Emir is an employee of Pfizer with stock and/or stock options, Employee of: Pfizer DOI: 10.1136/annrheumdis-2021-eular.2175

\section{POS0284 CLINICAL AND MRI COMPARISON OF ECCENTRIC VERSUS CONCENTRIC REHABILITATION IN SYMPTOMATIC KNEE OSTEOARTHRITIS: A PROSPECTIVE RANDOMIZED STUDY}

M. C. Trojani ${ }^{1}$, D. Bendahan ${ }^{2}$, F. Chorin ${ }^{3}$, P. Gerus ${ }^{4}$, V. Breuil ${ }^{5}$, S. Guis ${ }^{6}$, C. Roux ${ }^{7} .{ }^{1}$ Université cote d'azur, Rheumatology, Nice, France; ${ }^{2}$ Aix Marseille University, CNRS, Centre de Résonance Magnétique Biologique et Médicale (CRMBM), UMR 7339, Marseille, France; ${ }^{3}$ Université Cote d'Azur, LAMHESS laboratory, NICE, France; ${ }^{4}$ Université Cote d'Azur, LAMHESS laboratory, Nice, France; ${ }^{5}$ Université Cote d'Azur, Rheumatology, Nice, France; ${ }^{6}$ AixMarseille University, 13009, Marseille, France, CRMBM UMR CNRS 7339, Marseille, France: ${ }^{7}$ Université cote d'azur, Rheumatology, Nice, France

Background: Rehabilitation is at the center of the non-medical management of knee osteoarthritis. Concentric muscle strengthening is often preferred, while eccentric contractions play an important role in controlling knee flexion and knee stability and allow the development of a high level of strength with a low energy cost. But few studies have focused on these two technique

Objectives: To explore the effect of a 6-week-exercise program on function, pain and performance level in symptomatic knee osteoarthritis patients

Methods: An analysis was performed of the data from 60 individuals with symptomatic knee osteoarthritis who were included in the EXART study. The EXART study was a prospective, randomized controlled trial which included patients aged 40 to 85 with KL 2 or 3 responding to the American College of Rheumatology criteria. The first group benefitted from a 6 week eccentric rehabilitation program and the second group from a standard 6 week concentric rehabilitation program. The endpoints were the changes from baseline to week 6 in Western Ontario and McMaster Universities Osteoarthritis Index (WOMAC), pain, and performance measured with quadriceps power (PMax) and contraction strength (MMax). MRI muscle analysis was performed before and after physical activity. Results: Among 80 patients screened, 60 were included in the study and randomized. 25/30 subjects finished the program in the concentric group and 28/30 in the eccentric group. The mean population age was $74(+/-8)$, mean IMC 27.2 $(+/-4)$. The baseline total WOMAC mean was $49.2(+/-19)$, VAS pain $5.3(+/-2)$. At 6 weeks, both groups showed a significant improvement compared to baseline for the WOMAC without any difference between the two groups (mean difference evolution concentric $=7.5 \pm 11.9(p=p<0.0001)$; mean difference evolution eccentric $=4.5 \pm 1.9(p<0.0001)$; intergroup analysis $p=0.7)$. Similar significant results were found in the WOMAC function subscale. No difference between the groups appeared in VAS pain evolution $(p=0.7)$. The eccentric group showed a significant improvement in PMax and high speed MMax evolution ( $p=0.001$ and $p=0.002)$. This improvement was not found for the concentric group $(p=0.52$ and $p=0.27$ ). MRI showed a vastus medialis hypertrophy only in the eccentric group $(p=0.002)$. We did not observe any change in the fatty infiltration of the quadriceps on MRI.

Conclusion: Rehabilitation, whether eccentric or concentric, has a beneficial action on function and pain in symptomatic knee osteoarthritis whatever the methods. A gain in muscle performance and vastus medialis volume was found only with the eccentric rehabilitation. This study confirms the importance of any type of rehabilitation in knee osteoarthritis and raises the question of the relation between muscle gain/performance and function or pain

Acknowledgements: We would like to thank all the patients who took part in the study as well as all the members of the Fragile Platform of the Nice University Hospital.

Disclosure of Interests: None declared

DOI: 10.1136/annrheumdis-2021-eular.3164

\section{POS0285 ARE RACIAL DISPARITIES IN REVISION TKA OUTCOMES ASSOCIATED WITH HOSPITAL OR SURGEON VOLUME?}

S. Mirza ${ }^{1}$, S. Goodman ${ }^{2,3}$, Y. Zhang ${ }^{2}$, H. Do ${ }^{2}$, B. Mehta ${ }^{2,3}$, S. Lyman ${ }^{2}$, L. A. Mandl ${ }^{2,3}$, M. Figgie ${ }^{4}$, M. Parks ${ }^{4}$, L. Russell ${ }^{2,3}$, A. Bass ${ }^{2,3}{ }^{1}$ Touro College of Osteopathic Medicine, Medical School, New York, United States of America; ${ }^{2}$ Hospital for Special Surgery, Medicine, New York, United States of America; ${ }^{3}$ Weill Cornell Medical Center, Medicine, New York, United States of America; ${ }^{4}$ Hospital for Special Surgery, Orthopedic Surgery, New York, United States of America

Background: Total knee arthroplasty (TKA) outcomes are linked to surgical volume, ${ }^{1}$ despite the increase in TKA utilization, racial disparities in TKA outcomes persist. Blacks in the US are at a higher risk of aseptic revision of TKA (R-TKA) when compared to Whites, yet the reasons for this are not understood.

Objectives: The objective of this study is to examine the relationship between hospital and surgeon annual TKA volume and R-TKA outcomes by race.

Methods: This is an observational cohort study. New York Statewide Planning and Research Cooperative System data for 2004 - 2013 was used to identify patients who underwent primary TKA. Data through 2015 was used to identify R-TKA within 2 years of the index TKA. Hospital characteristics were obtained from the AHA Annual Survey. Surgeon data was collected from New York State Education Department and New York State Physician Profile. Surgeon annual TKA volume was categorized based on cutoffs established by Wilson et $\mathrm{al}^{1}$ as $</=12,13-59,60-145$ or $>/=146$, and hospital TKA volume as $</=89,90-235,236-644$ and $>/=645$. We calculated the odds of R-TKA in Whites and Blacks separately and generated crude odds ratios (OR) comparing Blacks to Whites to examine trends across volume categories. A multivariable logistic regression model adjusted for known R-TKA risk factors was also performed.

Results: A total of 163,576 patients were included. Mean (SD) age was 66.4 (10.4) years, 107,233 (65.6\%) were female, 124,277 (76.6\%) were White and 15,990 (9.8\%) were Black. 2925 patients underwent aseptic R-TKA. In logistic regression analysis, Blacks had a higher risk of R-TKA (OR 1.42, $95 \% \mathrm{Cl} 1.26$ 1.6) (Table 1). Risk of R-TKA was also higher when surgeon annual volume was $</=12$ (OR $1.5,95 \% \mathrm{Cl} 1.25-1.8$ ) or $13-59$ (OR $1.16,95 \% \mathrm{Cl} 1.04-1.29)$ TKA compared to the highest volume surgeons $(>/=146)$. Patients who had surgery at a hospital with annual volume of 236-634 TKA were less likely to undergo R-TKA compared to the highest volume hospitals $(>/=645)$ (OR $0.88,95 \% \mathrm{C}$ 0.79-0.98). Other risk factors for R-TKA were younger age and worker's compensation, while patients with inflammatory arthritis had a lower risk. Figures $1 \mathrm{~A}$ and $1 \mathrm{~B}$ show the odds of R-TKA in Whites and Blacks, respectively, by hospital and surgeon volume. Figure $1 \mathrm{C}$ shows the crude $\mathrm{OR}$ for Blacks to Whites for each category pair. The OR ranged from 0.9 to 2.5 , with the largest disparity found in patients who have TKA performed by surgeons with 60-145 annual TKA volume at the highest volume hospitals $(>/=645)$.

Conclusion: Patients having TKA by a surgeon performing $<60$ TKA per year have higher risk of R-TKA. Racial disparities in R-TKA risk are highest for TKA by surgeons performing 60-145 TKA per year at hospitals performing $>/=645$ TKA per year. Future studies should examine factors, such as whether trainees are involved the surgery, that may vary based on social determines of health, such as patient race and payor.

\section{REFERENCES:}

[1] Wilson S. et al Meaningful thresholds for the volume-outcome relationship in total knee arthroplasty. Journal of bone and joint surgery. 2016 98:1683 